\title{
Clinical Characteristics of Japanese Type 2 Diabetic Patients Responsive to Sitagliptin
}

\author{
Kouichi Inukai1 ${ }^{*}$, Takumi Hirata ${ }^{2}$, Takashi Sumita ${ }^{3}$, Masaki Watanabe $^{4}$, Yuichi Ikegami $^{3}$, \\ Daisuke Ito $^{3}$, Susumu Kurihara ${ }^{3}$, Nobuyuki Yasukawa ${ }^{3}$, Jiro Morimoto5, Nobuki Takata6, \\ Kenta Kanazawa6, Tamotsu Neda ${ }^{3}$, Yoshikazu Sumitani' ${ }^{1}$, Kiyoaki Inoue ${ }^{3}$, Yuichi Noguchi' \\ Toshio Hosaka ${ }^{3}$, Hitoshi Ishida ${ }^{1}$, Shigehiro Katayama ${ }^{3}$ \\ ${ }^{1}$ Third Department of Internal Medicine, Division of Diabetes, Endocrinology and Metabolism, Kyorin University \\ School of Medicine, Tokyo, Japan \\ ${ }^{2}$ Foundation for Biomedical Research and Innovation, Hyogo, Japan \\ ${ }^{3}$ Division of Endocrinology and Diabetes, Saitama Medical University, Saitama, Japan \\ ${ }^{4}$ Watanabe Medical Clinic, Hiroshima, Japan \\ ${ }^{5}$ Department of Internal Medicine, Saitama Social Insurance Hospital, Saitama, Japan \\ ${ }^{6}$ Sakado Central Hospital, Saitama, Japan \\ Email: ${ }^{*}$ kinukai@ks.kyorin-u.ac.jp
}

Received 10 May 2014; revised 8 June 2014; accepted 3 July 2014

Copyright ( 2014 by authors and Scientific Research Publishing Inc.

This work is licensed under the Creative Commons Attribution International License (CC BY).

http://creativecommons.org/licenses/by/4.0/

(c) (i) Open Access

\section{Abstract}

Japanese type 2 diabetic patients were treated with sitagliptin to evaluate the efficacy of this agent, and also to investigate the clinical characteristics of those who responded to sitagliptin. In total, 1001 diabetic patients, inadequately controlled (HbA1c $\geq 6.5 \%$ ) with oral hypoglycemic agents (OHA) other than DPP-4 inhibitors or with diet and exercise only, were enrolled. We added 50mg of sitagliptin to the therapeutic regimens of 410 patients including 68 OHA naïve patients, while the other 591 patients were switched from a single OHA to $50 \mathrm{mg}$ of sitagliptin. After 6 months, glycemic control was significantly improved due to both reduced insulin resistance, as demonstrated by a significant HOMA-R reduction, and recovery of pancreatic $\beta$ cell function, as assessed by HOMA- $\beta$ and the proinsulin/insulin (PI/I) ratio. In the bivariable analysis, a good response, defined as an HbA1c reduction during the 6 months of at least $0.9 \%$, was associated with high HbA1c and PI/I at baseline and combination treatments with sulfonylurea, biguanide and $\alpha$-glucosidase inhibitors, but not with obesity. On the other hand, in the multivariable regression analysis, only high baseline HbA1c and combination treatment with an $\alpha$-glucosidase inhibitor were significantly associated with a good response to sitagliptin. In patients with type 2 diabetes, the addition of sitagliptin or switching from another OHA to this agent achieved an HbA1c reduction without overloading $\beta$ cells. In particular, we suggest that a good response to sitagliptin can be expected when this agent is combined with an $\alpha$-glucosidase inhibitor (UMIN No. \#000014157).

\footnotetext{
${ }^{*}$ Corresponding author.

How to cite this paper: Inukai, K., Hirata, T., Sumita, T., Watanabe, M., Ikegami, Y., Ito, D., Kurihara, S., Yasukawa, N., Morimoto, J., Takata, N., Kanazawa, K., Neda, T., Sumitani, Y., Inoue, K., Noguchi, Y., Hosaka, T., Ishida, H. and Katayama, S. (2014) Clinical Characteristics of Japanese Type 2 Diabetic Patients Responsive to Sitagliptin. Journal of Diabetes Mellitus, 4, 172-178. http://dx.doi.org/10.4236/jdm.2014.43025
} 


\section{Keywords}

\section{Sitagliptin, Type 2 Diabetes, DPP-4 Inhibitors}

\section{Introduction}

Dipeptidyl peptidase-4 (DPP-4) inhibitors stimulate glucose-dependent insulin secretion by increasing the activity of endogenous glucagon-like peptide (GLP-1) and glucose-dependent insulinotropic polypeptides (GIP), which are hormones released from intestinal cells in response to carbohydrate absorption [1]. Thus, DPP-4 inhibitors can improve both fasting and postprandial glucose levels without hypoglycemic episodes, which provides a definite benefit as compared with conventional insulin secretagogues, i.e. sulfonylureas (SU) [2] [3]. At present, seven DPP-4 inhibitors are available in Japan: sitagliptin, vildagliptin, alogliptin, linagliptin, teneligliptin, anagliptin and saxagliptin [4]-[9]. Sitagliptin, the first of these DPP-4 inhibitors, was approved at the end of 2010, and is now widely available in Japan. Despite being the most commonly used DPP-4 inhibitor in Japan; predictive factors of the HbA1c-lowering effects of sitagliptin have not yet been elucidated. Herein, we investigated the efficacy of sitagliptin and also patient characteristics related to a good response to sitagliptin at multiple medical centers. Six months after the initiation of $50 \mathrm{mg}$ sitagliptin treatment, glycemic control was significantly improved. The only baseline characteristics significantly associated with a good response to sitagliptin in the multivariable regression analysis were high HbA1c and combination therapy with an $\alpha$-glucosidase inhibitor $(\alpha$-GI). Our results provide guidance for the optimal use of sitagliptin for managing patients with type 2 diabetes.

\section{Materials and Methods}

\subsection{Study Design and Methods}

After obtaining approval from the Institutional Ethics Review Committees of the participating hospitals, a total of 1001 type 2 diabetic patients, inadequately controlled (HbA1c $\geq 6.5 \%$ ) with oral hypoglycemic agents (OHA) other than DPP-4 inhibitors or with diet and exercise only, were enrolled in this multi-center, open-label, interventional study. We added $50 \mathrm{mg}$ of sitagliptin to the treatment regimens of 410 patients with poor glycemic control (HbA1c $\geq 6.5 \%$ ) including 68 OHA naïve patients. When a $50 \mathrm{mg}$ dose of sitagliptin was added to the regimens of diabetic patients already treated with more than $2 \mathrm{mg}$ of glimepiride, the glimepiride dose was reduced to $2 \mathrm{mg}$ or less, according to the JDS (Japan Diabetes Association) recommendation, in order to avoid severe hypoglycemia. Thus, these 410 patients were divided into 2 groups and analyzed individually in Studies 1 and 2. In Study 1, a $50 \mathrm{mg}$ dose of sitagliptin was simply added to the regimens of 249 patients, treated with 2 mg or less of glimepiride (study 1). The other 161 patients, who were treated with more than $2 \mathrm{mg}$ of glimepiride, received 50mg of sitagliptin with a reduction in the glimepiride dose to less than $2 \mathrm{mg}$ (study 2). Another 591 patients were switched from a single OHA to $50 \mathrm{mg}$ of sitagliptin (study 3 ). When the patients were treated with more than 2 OHA, the physicians in charge decided which OHA was switched to sitagliptin, based on their clinical experience. We also relied on physicians in charge to decide which study the patients would be enrolled in, i.e. add-on therapy (study 1 or 2) or replacement therapy (study 3). However, patients with relatively poor glycemic control tended to be enrolled in study 1 or 2, because these patients needed to be treated with an additional OHA to improve their glycemic control. In practice, the mean baseline HbA1c levels were significantly higher in patients enrolled in studies 1 and 2 than in those participating in study $3(7.9 \% \pm 0.6 \%, 7.3 \%+0.8 \%$, respectively). Except for HbA1c and fasting plasma glucose (FPG), there were no significant differences in baseline characteristics among these three groups. Overall, patients with impaired hepatic function (serum AST/ALT > 40) or renal function (serum creatinine > 1.5) were excluded. Treatments with other drugs including OHA remained unchanged throughout the six-month study period. The following parameters were measured employing standard laboratory techniques at baseline and at 6 months after the initiation of treatment; FPG, fasting insulin (IRI) and HbA1C (NGSP). In addition, indexes that are considered to reflect insulin resistance (HOMA-R = FPG X IRI/405) and $\beta$-cell function (HOMA- $\beta=360$ X IRI/FPG-63) were calculated. At baseline and at the initiation of this study, we also measured 1,5-AG, serum total adiponectin and the proinsulin/insulin 
(PI/I) ratio. These measurements were all carried out by SRL Inc. (Tokyo, Japan). All patients gave written informed consent prior to participating in these studies.

\subsection{Statistical Analysis}

Data are presented as means \pm standard deviation for continuous variables. We used the Wilcoxon signed-rank test to compare variables between 0 and 6 months after administration of sitagliptin. We analyzed factors associated with a good response (defined as an HbA1c reduction during the 6 months of at least $0.9 \%$ ) employing univariable and multivariable logistic regression analyses. A $P$ value of $<0.05$ was considered statistically significant. All analyses were performed using STATA SE 11 data analysis and statistical software (Stata Corp LP, College Station, TX, USA).

\section{Results}

Changes in the main parameters in Studies 1 and 2 are shown in Table 1. In total, 25 patients dropped out because of drug side effects or failure to return for hospital visits. Glycemic control was markedly improved, in terms of both HbA1c and FPG, in both Study 1 and Study 2. Particularly, HbA1c was reduced by $0.7 \%$, despite the glimepiride dose being decreased from $2.6 \pm 0.1 \mathrm{mg}$ to $1.2 \pm 0.1 \mathrm{mg}$ in Study 2. In addition to the improvements in both HOMA-R and HOMA- $\beta$, the PI/I ratio also improved, suggesting that the recovery of $\beta$ cell function was not due to forced overloading, and that there had actually been a load reduction. The 1,5-AG level was also markedly increased, indicating glycemic fluctuation to be attenuated. Study 3, which examined the effects of switching agents, is summarized, with average doses and the number of patients, in Table 2. The average doses of each of the OHA classes were calculated as follows; $40 \mathrm{mg}$ of gliclazide was equivalent to $1 \mathrm{mg}$ of glimepiride, $0.6 \mathrm{mg}$ of voglibose was equivalent to $50 \mathrm{mg}$ of miglitol, and $90 \mathrm{mg}$ of nateglinide was equivalent to $10 \mathrm{mg}$ of mitiglinide. As a result, small doses of SU and biguanides (BG), and standard doses of $\alpha$-GI, glinides and thiazolidinediones were switched to $50 \mathrm{mg}$ of sitagliptin. Table 3 shows the results of study 3 . Glycemic control was slightly but significantly improved, whereas HOMA- $\beta$ was decreased.

Table 4 shows single or multiple correlations between each parameter at baseline and effectiveness, defined as a more than $0.9 \% \mathrm{HbA} 1 \mathrm{c}$ reduction at the end of the study. Among single correlations, high HbA1c, low HOMA- $\beta$, high PI/I ratio, high 1,5-AG and combination therapy with SU, $\alpha$-GI, or BG were associated with the effectiveness of sitagliptin. For multivariable regression analysis, we adopted two models (Models 1 and 2), containing HOMA- $\beta$ or the PI/I ratio, respectively, because these two parameters are interdependent. In both Model 1 and Model 2, high HbA1c and combination therapy with an $\alpha$-GI were still positively associated with the effectiveness of sitagliptin, which provides information on the optimal use of DPP-4 inhibitors in type 2 diabetic patients.

\section{Discussion}

In the present study, we investigated the effect of administering $50 \mathrm{mg}$ of sitagliptin, which is a standard starting dosage for this medication in Japan. However, outside of Japan, double this dose, i.e. $100 \mathrm{mg}$ of sitagliptin, is generally used [10]. The dose-ranging efficacy of sitagliptin was tested in a previous study [11], in which no differences in HbA1c were observed among sitagliptin doses of $50 \mathrm{mg}, 100 \mathrm{mg}$, and $200 \mathrm{mg}$ in Japanese patients with type 2 diabetes mellitus. According to these results, the $50 \mathrm{mg}$ dose of sitagliptin was adopted as the standard for Japanese patients. Thus, Japanese patients are considered to be more responsive to sitagliptin than non-Japanese patients. A meta-analysis revealed that, despite the smaller sitagliptin dose of only $50 \mathrm{mg}$, a greater HbA1c reduction ( $-0.99 \%$ versus placebo) was observed in Japanese than in non-Japanese patients [12]. A marked HbA1c reduction ( $-1.0 \%$ versus baseline) was also observed in this study with add-on therapy, which is consistent with the previous results. Despite GLP-1 levels not being decreased in Japanese patients [13], a good response to sitagliptin was still demonstrated. One possible explanation is that the body mass index (BMI) is generally lower in Japanese than in non-Japanese patients [12]. DPP-4 was previously reported to be one of the adipokines, playing a role in the induction of insulin resistance and providing a link between obesity and type 2 diabetes [14]. In addition, non-esterified fatty acids (NEFA) were demonstrated to be involved in reducing incretin signaling [15] or incretin secretion from the small intestine [16]. Thus, in obese patients, whose serum levels of DPP-4 or NEFA are predicted to be higher than those of normal weight subjects, the glucose-lowering 
Table 1. Clinical parameters before the study (Baseline) and changes after 6 months (Endpoints) in Studies 1 and $2 .{ }^{*} P$ value $<0.05$.

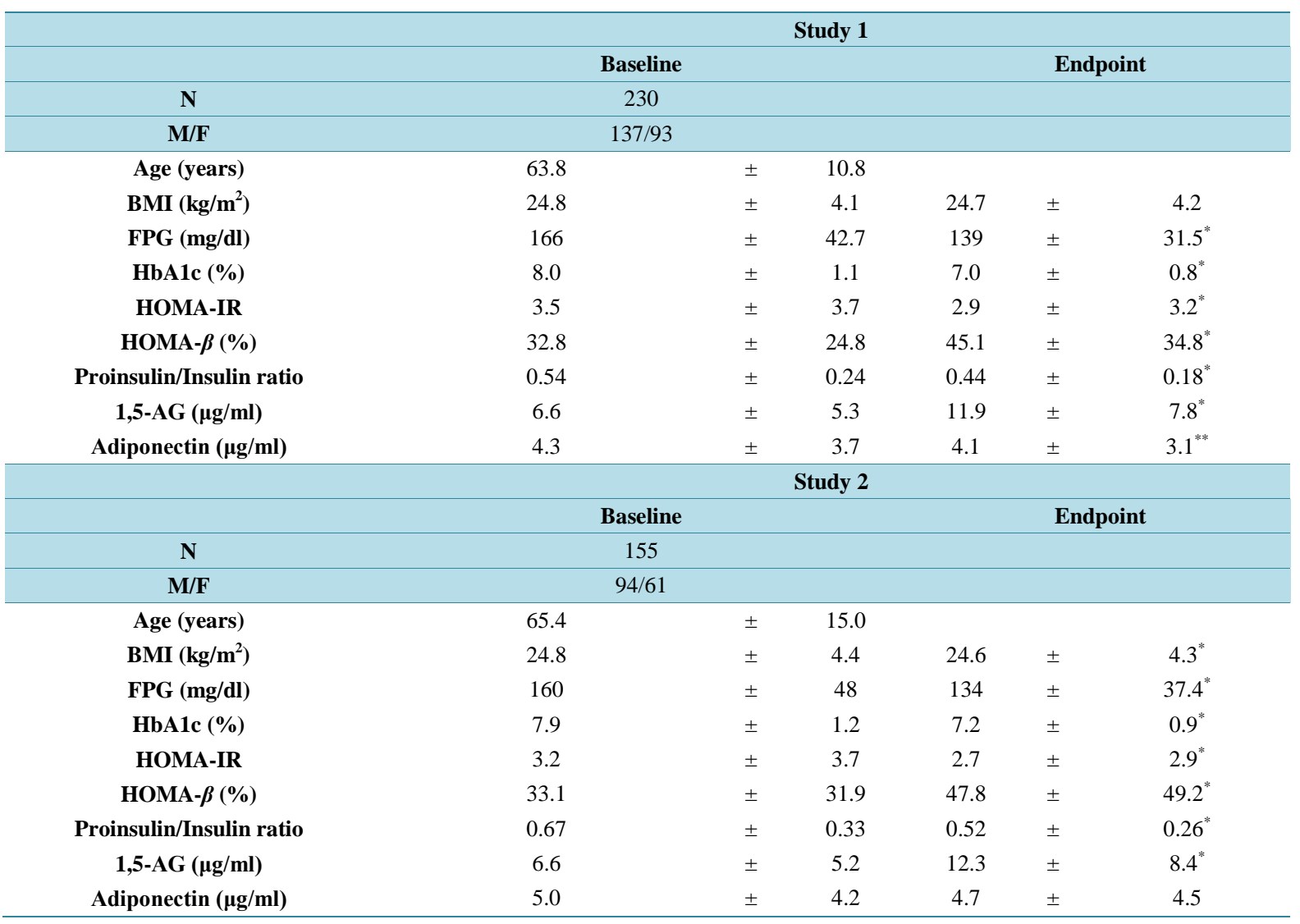

Table 2. Number, mean dosage and name of OHA switched to sitagliptin in Study 3.

\begin{tabular}{cccc}
\hline OHA & Major Agent & $\mathrm{n}$ & dose (mg) \\
\hline Sulfonylurea & glimepiride/gliclazide & 94 & 0.7 (glimepiride) \\
$\alpha$-Glucosidase inhibitor & miglitol/voglibose/acarbose & 235 & 784 (miglitol) \\
Biguanide & metformin & 75 & 28.5 (mitiglinide) \\
Glinide & mitiglinide/nateglinide & 69 & 20.2 (pioglitazone) \\
Thiazolidinedione & pioglitazone & (metformin) & \\
\hline
\end{tabular}

Table 3. Clinical parameters before the study (Baseline) and changes after 6 months (Endpoints) in Study $3 .{ }^{*} P$ value $<0.05$.

\begin{tabular}{|c|c|c|c|c|c|c|}
\hline & \multicolumn{6}{|c|}{ Study 3} \\
\hline & \multicolumn{3}{|c|}{ Baseline } & \multicolumn{3}{|c|}{ Endpoint } \\
\hline $\mathrm{N}$ & \multicolumn{3}{|c|}{551} & & & \\
\hline $\mathrm{M} / \mathrm{F}$ & \multicolumn{3}{|c|}{$345 / 206$} & & & \\
\hline Age (years) & 63.4 & \pm & 10.3 & & & \\
\hline $\mathrm{BMI}\left(\mathrm{kg} / \mathrm{m}^{2}\right)$ & 24.4 & \pm & 4.1 & 24.3 & \pm & $4.2^{*}$ \\
\hline FPG (mg/dl) & 148 & \pm & 36.4 & 140 & \pm & $40.6^{*}$ \\
\hline HbA1c (\%) & 7.3 & \pm & 0.8 & 7.1 & \pm & $0.9^{*}$ \\
\hline HOMA-IR & 2.9 & \pm & 2.9 & 3.0 & \pm & 3.9 \\
\hline НОМА- $\beta(\%)$ & 37.7 & \pm & 37.1 & 37.0 & \pm & $81.8^{*}$ \\
\hline Proinsulin/Insulin ratio & 0.56 & \pm & 0.34 & 0.54 & \pm & 0.29 \\
\hline $1,5-\mathrm{AG}(\mu \mathrm{g} / \mathrm{ml})$ & 9.9 & \pm & 7.1 & 11.0 & \pm & $7.0^{*}$ \\
\hline Adiponectin $(\mu \mathrm{g} / \mathrm{ml})$ & 5.0 & \pm & 4.7 & 3.9 & \pm & $3.4^{*}$ \\
\hline
\end{tabular}


Table 4. Bivariable and multivariable analyses, investigating the association of each parameter. with reduced HbA1c (more than $0.9 \%) .{ }^{*} P$ value $<0.05$.

\begin{tabular}{|c|c|c|c|c|c|c|}
\hline & Bivariable & & Multivariable & & & \\
\hline & & & Model 1 & & Model 2 & \\
\hline Factor & OR & $95 \% \mathrm{CI}$ & OR & $95 \% \mathrm{CI}$ & OR & $95 \%$ CI \\
\hline Sex (Male) & 1.34 & $0.99-1.82$ & & & & \\
\hline Age & 1.00 & $0.99-1.02$ & & & & \\
\hline BMI & 1.11 & $0.83-1.50$ & & & & \\
\hline HbA1c & $4.06^{*}$ & $3.28-5.04$ & $3.86^{*}$ & $2.41-6.17$ & $3.88^{*}$ & $2.44-6.17$ \\
\hline $\operatorname{HOMA}^{\dagger}{ }^{\dagger}$ & 1.18 & $0.93-1.49$ & & & & \\
\hline HOMA- $\beta^{\ddagger}$ & $0.49^{*}$ & $0.38-0.65$ & 0.83 & $0.53-1.29$ & & \\
\hline Proinsulin/Insulin ratio ${ }^{\dagger}$ & $2.45^{*}$ & $1.50-4.00$ & & & 1.63 & $0.86-3.11$ \\
\hline $1,5-\mathrm{AG}^{\dagger}$ & $0.32^{*}$ & $0.24-0.44$ & 0.91 & $0.54-1.55$ & 0.89 & $0.53-1.50$ \\
\hline Adiponectin $^{\dagger}$ & 0.86 & $0.65-1.13$ & & & & \\
\hline \multicolumn{7}{|l|}{ Combination with } \\
\hline Sulfonylurea & $2.41^{*}$ & $1.79-3.26$ & 1.13 & $0.60-2.15$ & 1.15 & $0.61-2.18$ \\
\hline$\alpha$-Glucosidase inhibitor & $3.26^{*}$ & $2.09-5.08$ & $3.25^{*}$ & $1.40-7.53$ & $3.22^{*}$ & $1.38-7.50$ \\
\hline Biguanide & $1.39^{* *}$ & $1.04-1.85$ & 1.29 & $0.71-2.34$ & 1.25 & $0.69-2.28$ \\
\hline Glinide & 1.76 & $0.57-5.43$ & & & & \\
\hline Thiazolidinedione & 1.45 & $0.93-2.28$ & & & & \\
\hline
\end{tabular}

effect of DPP-4 inhibitors may be attenuated.

In addition to the prominent HbA1c reduction, marked recovery of the PI/I ratio was also observed in the present study (study 1). These results reflect the protective effect of GLP-1 on beta cells via alleviation of endoplasmic reticulum stress [17]. Though a similar effect was previously reported in non-Japanese patients [18], our report is, to our knowledge, the first to document a PI/I ratio reduction in response to taking a DPP-4 inhibitor in Japanese patients with type 2 diabetes. The PI/I ratio reduction was greater in Japanese than in non-Japanese patients (19\% and $12 \%$ reductions, respectively). These observations may explain the beneficial effects of DPP-4 inhibitors in Japanese patients whose beta cell function is exhausted judging from their low of HOMA- $\beta$ levels. Taking into consideration that a greater PI/I ratio reduction (22\%) was obtained when the SU dosage was reduced in Study 2, add-on therapy with 50mg of sitagliptin with a reduced SU dosage may confer a major protective effect on beta cells in Japanese diabetic patients.

Several studies have reported factors contributing to the efficacy of sitagliptin in Japanese diabetic patients [19]-[21]. In these studies, low BMI and high HbA1c were common predictive factors for HbA1c reduction. Though high baseline HbA1c is consistent with our present data, why low BMI was not associated with the efficacy of sitagliptin is unknown. The method of sitagliptin treatment, i.e. add-on or switching therapy, the study scale and the study duration may have influenced the relevance of obesity to the HbA1c reduction. Instead of BMI, our study indicated diminished beta cell function, i.e. low HOMA- $\beta$ and a high PI/I ratio, to predict HbA1c reduction. Though DPP-4 inhibitors ameliorate insulin resistance (HOMA-R) probably by inhibiting glucagon secretion [11], DPP-4 inhibitors belong to a class of insulin secretagogues. Our results are considered to essentially reflect the original characteristics of DPP-4 inhibitors, which are known to improve glycemic control by enhancing insulin secretion, in diabetic patients with decreased beta cell function.

It has yet to be determined which OHA would most effectively reduce HbA1c when combined with DPP-4 inhibitors. Among possible candidates, combination therapy with metformin was reported to be beneficial, because DPP-4 inhibitors and metformin have complementary mechanisms of action and additive effects with respect to increasing the concentrations of active GLP-1 in plasma [22]. Inhibition of DPP-4 activity by metformin is speculated to be the mechanism by which metformin enhances GLP-1 [23]. Though our data also support compatibility between DPP-4 inhibitors and metformin, combination therapy with an $\alpha$-GI showed a stronger relationship with HbA1c reduction in our multivariable regression analysis. Therapy combining sitagliptin with miglitol, in which the ability of $\alpha$-GI to enhance GLP-1 secretion was discovered, was previously reported [24]. Our study is the first to clinically demonstrate the compatibility between a DPP-4 inhibitor, sitagliptin, and an $\alpha$-GI in Japanese patients with type 2 diabetes. 


\section{Conclusion}

In conclusion, the addition of sitagliptin to an already administered regimen or switching from another OHA resulted in reduced HbA1c due to recovery of $\beta$-cell function. In addition, we suggest that a good response to sitagliptin can be predicted when this agent is used in the combination with an $\alpha$-GI. These results provide useful insights for managing diabetic patients by using DPP-4 inhibitors.

\section{Acknowledgements}

None of the authors has any potential conflicts of interest associated with this research.

\section{References}

[1] Tarrani, A.A. and Bailey, C.J. (2011) Management of Type 2 Diabetes: New and Future Developments in Treatment. Lancet, 378, 182-197. http://dx.doi.org/10.1016/S0140-6736(11)60207-9

[2] Arai, K., Matoba, K., Hirao, K., Matsuba, I., Takai, M., Takeda, H., Kanamori, A., Yamauchi, M., Mori, H. and Terauchi, Y. (2010) Present Status of Sulfonylurea Treatment for Type 2 Diabetes in Japan: Second Report of a CrossSectional Survey of 15,652 Patients. Endocrine Journal, 57, 499-507. http://dx.doi.org/10.1507/endocrj.K09E-366

[3] Onuma, H., Inukai, K., Watanabe, M., Sumitani, Y., Hosaka, T. and Ishida, H. (2014) Effects of Long-Term Monotherapy with Glimepiride vs Glibenclamide on Glycemic Control and Macrovascular Events in Japanese Type 2 Diabetic Patients. Journal of Diabetes Mellitus, 4, 33-37. http://dx.doi.org/10.4236/jdm.2014.41006

[4] Schweizer, A., Dejager, S., Foley, J.E. and Kothny, W. (2011) Assessing the General Safety and Tolerability of Vildagliptin: Value of Pooled Analyses from a Large Safety Database versus Evaluation of Individual Studies. Vascular Health and Risk Management, 7, 49-57. http://dx.doi.org/10.2147/VHRM.S16925

[5] Pratley, R.E. (2009) Alogliptin: A New, Highly Selective Dipeptidyl Peptidase-4 Inhibitor for the Treatment of Type 2 Diabetes. Expert Opinion on Pharmacotherapy, 10, 503-512. http://dx.doi.org/10.1517/14656560802694713

[6] Araki, E., Kawamori, R., Inagaki, N., Watada, H., Hayashi, N., Horie, Y., Sarashina, A., Thiemann, S., Eynatten, M., Dugi, K. and Woerle, H.J. (2011) Long-Term Safety of Linagliptin Monotherapy in Japanese Patients with Type 2 Diabetes. Diabetes, Obesity and Metabolism, 13, 542-550.

[7] Eto, T., Inoue, S. and Kadowaki, T. (2012) Effects of Once-Daily Teneligliptin on 24-h Blood Glucose Control and Safety in Japanese Patients with Type 2 Diabetes Mellitus: A 4-Week, Randomized, Double-Blind, Placebo-Controlled Trial. Diabetes, Obesity and Metabolism, 14, 1040-1046. http://dx.doi.org/10.1111/j.1463-1326.2012.01662.x

[8] Ervinna, N., Mita, T., Yasunari, E., Azuma, K., Tanaka, R., Fujimura, S., Sukmawati, D., Nomiyama, T., Kanazawa, A., Kawamori, R., Fujitani, Y. and Watada, H. (2013) Anagliptin, a DPP-4 Inhibitor, Suppresses Proliferation of Vascular Smooth Muscles and Monocyte Inflammatory Reaction and Attenuates Atherosclerosis in Male Apo E-Deficient Mice. Endocrinology, 154, 1260-1270. http://dx.doi.org/10.1210/en.2012-1855

[9] Gerrald, K.R., Van Scoyoc, E., Wines, R.C., Runge, T. and Jonas, D.E. (2012) Saxagliptin and Sitagliptin in Adult Patients with Type 2 Diabetes: A Systematic Review and Meta-Analysis. Diabetes, Obesity and Metabolism, 14, 481-492. http://dx.doi.org/10.1111/j.1463-1326.2011.01540.x

[10] Williams-Herman, D., Engel, S.S., Round, E., Johnson, J., Golm, G.T., Guo, H., Musser, B.J., Davies, M.J., Kaufman, K.D. and Goldstein, B.J. (2010) Safety and Tolerability of Sitagliptin in Clinical Studies: A Pooled Analysis of Data from 10,246 Patients with Type 2 Diabetes. BMC Endocrine Disorders, 10, 7. http://dx.doi.org/10.1186/1472-6823-10-7

[11] Iwamoto, Y., Taniguchi, T., Nonaka, K., Okamoto, T., Okuyama, K., Arjona Ferreira, J.C. and Amatruda, J. (2010) Dose-Ranging Efficacy of Sitagliptin, a Dipeptidyl Peptidase-4 Inhibitor, in Japanese Patients with Type 2 Diabetes Mellitus. Endocrine Journal, 57, 383-394. http://dx.doi.org/10.1186/1472-6823-10-7

[12] Park, H., Park, C., Kim, Y. and Rascati, K.L. (2012) Efficacy and Safety of Dipeptidyl Peptidase-4 Inhibitors in Type 2 Diabetes: Meta-Analysis. Annals of Pharmacotherapy, 46, 1453-1469. http://dx.doi.org/10.1345/aph.1R041

[13] Lee, S., Yabe, D., Nohtomi, K., Takada, M., Morita, R., Seino, Y. and Hirano, T. (2010) Intact Glucagon-Like Peptide-1 Levels Are Not Decreased in Japanese Patients with Type 2 Diabetes. Endocrine Journal, 57, 119-126. http://dx.doi.org/10.1507/endocri.K09E-269

[14] Lamers, D., Famulla, S., Wronkowitz, N., Hartwig, S., Lehr, S., Ouwens, D.M., Eckardt, K., Kaufman, J.M., Ryden, M., Müller, S., Hanisch, F.G., Ruige, J., Arner, P., Sell, H. and Eckel, J. (2011) Dipeptidyl Peptidase 4 Is a Novel Adipokine Potentially Linking Obesity to the Metabolic Syndrome. Diabetes, 60, 1917-1925. http://dx.doi.org/10.2337/db10-1707

[15] Kang, Z.F., Deng, Y., Zhou, Y., Fan, R.R., Chan, J.C., Laybutt, D.R., Luzuriaga, J. and Xu, G. (2013) Pharmacological 
Reduction of NEFA Restores the Efficacy of Incretin-Based Therapies through GLP-1 Receptor Signalling in the Beta Cell in Mouse Models of Diabetes. Diabetologia, 56, 423-433. http://dx.doi.org/10.1007/s00125-012-2776-x

[16] Ranganath, L.R., Beety, J.M. and Morgan, L.M. (1996) Attenuated GLP-1 Secretion in Obesity: Cause or Consequence? Gut, 38, 916-919. http://dx.doi.org/10.1136/gut.38.6.916

[17] Yusta, B., Baggio, L.L., Estall, J.L., Koehler, J.A., Holland, D.P., Li, H., Pipeleers, D., Ling, Z. and Drucker, D.J. (2006) GLP-1 Receptor Activation Improves Beta Cell Function and survival Following Induction of Endoplasmic Reticulum Stress. Cell Metabolism, 4, 391-406. http://dx.doi.org/10.1016/j.cmet.2006.10.001

[18] Raz, I., Hanefeld, M., Xu, L., Caria, C., Williams-Herman, D. and Khatami, H. (2006) Sitagliptin Study 023 Group, Efficacy and Safety of the Dipeptidyl Peptidase-4 Inhibitor Sitagliptin as Monotherapy in Patients with type 2 Diabetes Mellitus. Diabetologia, 49, 2564-2571. http://dx.doi.org/10.1007/s00125-006-0416-z

[19] F-Wind and Chikushi-JRN Group (2012) Contributing Factors Related to Efficacy of the Dipeptidyl Peptidase-4 Inhibitor Sitagliptin in Japanese Patients with Type 2 Diabetes. Diabetes Research and Clinical Practice, 95, e27-e28. http://dx.doi.org/10.1016/j.diabres.2011.08.016

[20] Maeda, H., Kubota, A., Tanaka, Y., Terauchi, Y. and Matsuba, I. (2012) The Safety, Efficacy and Predictors for HbA1c Reduction of Sitagliptin in the Treatment of Japanese Type 2 Didbetes. Diabetes Research and Clinical Practice, 95, e20-e22. http://dx.doi.org/10.1016/j.diabres.2011.10.011

[21] Bando, Y., Kanehara, H., Aoki, K., Hisada, A., Toya, D. and Nobuyoshi, T. (2012) Obesity May Attenuate the HbA1c-Lowering Effect of Sitagliptin in Japanese Type 2 Diabetic Patients. Journal of Diabetes Investigation, 3, 170174. http://dx.doi.org/10.1111/j.2040-1124.2011.00156.x

[22] Solis-Herrera, C., Triplitt, C., Garduno-Garcia, J.J., Adams, J., Defronzo, R.A. and Cersosimo, E. (2013) Mechanisms of Glucose Lowering of Dipeptidyl Peptidase-4 Inhibitor Sitagliptin When Used Alone or with Metformin in Type 2 Diabetes: A Double-Tracer Study. Diabetes Care, 36, 2756-2762. http://dx.doi.org/10.2337/dc12-2072

[23] Green, B.D., Irwin, N., Duffy, N.A., Gault, V.A., O’Harte, F.P. and Flatt, P.R. (2006) Inhibition of Dipeptidyl Peptidase-IV Activity by Metformin Enhances the Antidiabetic Effects of Glucagon-Like Peptide-1. European Journal of Pharmacology, 547, 192-199. http://dx.doi.org/10.1016/j.ejphar.2006.07.043

[24] Aoki, K., Masuda, K., Miyazaki, T., Togashi, Y. and Terauchi, Y. (2010) Effects of Miglitol, Sitagliptin or Their Combination on Plasma Glucose, Insulin and Incretin Levels in Non-Diabetic Men. Endocrine Journal, 57, 667-672. http://dx.doi.org/10.1507/endocrj.K10E-103 
Scientific Research Publishing (SCIRP) is one of the largest Open Access journal publishers. It is currently publishing more than 200 open access, online, peer-reviewed journals covering a wide range of academic disciplines. SCIRP serves the worldwide academic communities and contributes to the progress and application of science with its publication.

Other selected journals from SCIRP are listed as below. Submit your manuscript to us via either submit@scirp.org or Online Submission Portal.
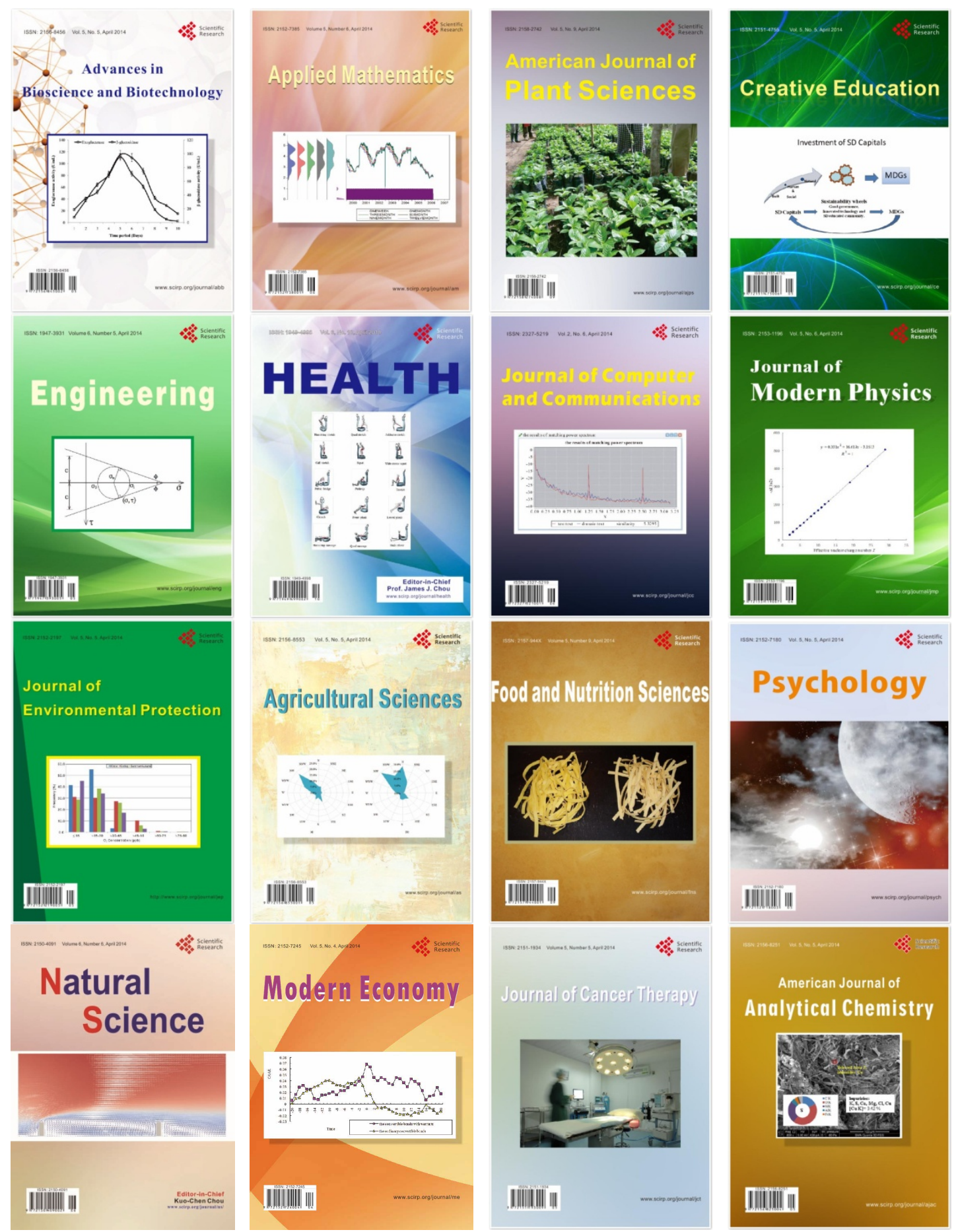